\title{
Active damping method for voltage source inverter-based distributed generator using multivariable finite-control-set model predictive control
}

Jonggrist Jongudomkarn, Warayut Kampeerawat

Department of Electrical Engineering, Khon Kaen University, Khon Kaen, Thailand

\begin{tabular}{l}
\hline \hline Article Info \\
\hline Article history: \\
Received Oct 26, 2020 \\
Revised Jan 20, 2021 \\
Accepted Feb 4, 2021
\end{tabular}

Keywords:

Active damping

Distributed power generations

Power quality

Predictive control

Voltage source inverter

\begin{abstract}
Despite its advantages, the $L C L$ filter can significantly distort the grid current and constitute a substantially more complex control issue for the gridconnected distributed generators (DGs). This paper presents an active damping approach to deal with the $L C L$ filter's oscillation for the finitecontrol-set model predictive control (FCS-MPC)-three-phase voltage source inverters (VSIs)-based DG. The new approaches use the multivariable control of the inverter side's filter current and capacitor voltage to suppress the $L C L$ filter resonance. The proposed method has been tested in steadystate and under grid voltage disturbances. The comparative study was also conducted with the existing virtual resistance active damping approaches for an FCS-MPC algorithm. The study validates the developed control schemes' superior performance and shows its ability to eliminate lower-order grid current harmonics and decrease sensitivity to grid voltage distortion.
\end{abstract}

This is an open access article under the CC BY-SA license.

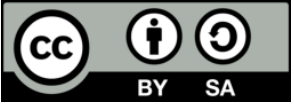

\section{Corresponding Author:}

Jonggrist Jongudomkarn

Department of Electrical Engineering, Faculty of Engineering, Khon Kaen University

123 Moo 16 Mittraphap Rd., Nai-Muang, Muang District, Khon Kaen 40002, Thailand

Email: jongjo@kku.ac.th

\section{INTRODUCTION}

The indiscriminate uses of fossil fuels to supply the indefinite demand of the world's energy have led to the rapid depletion of these non-renewable resources. Furthermore, their exploitation is also believed to be the leading cause of global warming and climate change [1]. One possible solution for this problem lies in using renewable energy sources (RES) such as wind and solar power. This is evidenced by the global capacity of wind turbines and solar cells, which are increasing annually. However, unlike conventional power generation, RESs usually require a topologically power-source-dependent power converter to deliver their power to the grid [2]-[4]. Such power-converter-based- generators are often operating in parallel with the utility distribution system near the loads. Hence, they are commonly referred to as "distributed generator (DG). In the past years, the finite control set (FCS-MPC) has cemented its place as an appealing alternative for controlling power converters, thanks to its versatile control scheme that enables easy inclusion of multiple control variables, restrictions, and nonlinearities [5]-[14]. Previously, several control algorithms based on FCS-MPC for DGs' applications have been proposed in the literature; for instance, a FCS-MPC strategy for a grid-connected 3L-NPC appears in [9]. A study on the FCS-MPC scheme for grid-connected VSIs was conducted in [10]. Additionally, other FCS-MPC-based methods for various power converters' topologies are proposed in [11]-[15]. Conclusively, the FCS-MPC scheme's benefits are presented, including simple implementation, straightforward control law, and fast dynamic response. The conventional FCS-MPC-based DG system, however, has been designed for a system with simple inductive filters ( $L$ filters) or inductive 
capacitance filters ( $L C$ filters), e.g., presented in [13]-[14]. On the other hand, to achieve sinusoidal grid currents with low distortions, an $L C L$ filter is an attractive alternative with relatively lower cost compared with conventional $L C$ filters [16]-[17]. Nevertheless, the third-order $L C L$ filter poses a substantially more complicated control issue as harmonics components close to the resonance's frequencies may be generated by the switching harmonics. Moreover, the average switching frequency of FCS-MPC is unfeasible to predetermine because it does not depend only on the sampling frequency and varies with the inverters operating point. To fulfill the grid code requirements, it is essential to reduce the total harmonic distortion (THD) under a certain threshold. Therefore, active damping (AD) methods are necessarily required for gridconnected DG applications with $L C L$ filters. According to the literature [17]-[21], scholars have proposed many damping approaches, mainly divided into passive damping and active damping. Passive damping is not a preferred method as it adds additional loss to the system and has low flexibility. On the other hand, extra attention must be paid while introducing active damping methods to FCS-MPC applications, as suggested in [17]-[19], because FCS-MPC produces variable switching frequencies. One of the preferred choices of active damping methods for FCS-MPC is the virtual resistance (VR) method. This scheme's essence is to add parallel virtual impedance at the filter capacitor to suppress the filter resonance. The advantage of this scheme is the high dynamic response of the power control. However, it utilizes a grid-following/ gridinjecting strategy and can only operate in the grid-connected operation. Furthermore, when harmonics distort the grid voltage, there is a chance that those harmonics will be unendingly fed back to the current reference through the VR current leading to increased distortion of the current waveforms.

Recently, several active damping solutions based on multivariable control for grid-connected rectifiers have been proposed [22]-[24]. The concept of FCS-MPC-based multivariable control can also be applied to grid-connected voltage source inverters (VSIs), as suggested in [13]-[15]. Nevertheless, in the referred works, the purpose of a filter current control inclusion was to achieve overcurrent prevention. Moreover, the study regarding the active damping ability of the multivariable FCS-MPC-based DG is still mostly uncovered. In this paper, a new feedback approach, including multivariable control of output filter's voltage and current based on FCS-MPC, are presented and compared with the well-known virtual-resistance active damping method. The proposed AD's approach helps to maintain the sinusoidal waveforms of the inverter's side filter current and the capacitor voltage through the real-time control of these two variables in $\alpha \beta$-coordination. Meaning, unlike the VR-method, there is no distorted current reference feeding back to the system during grid disturbances. Hence, the proposed control is more robust against grid voltage distortion. With the voltage and current regulation, the proposed scheme can also operate in both grid-connected and stand-alone operations. Simultaneously, the flexibility of the multivariable control allows easy integration of the proposed AD scheme with different control algorithms. This is very beneficial for DG applications, where various conditions need to be carefully observed simultaneously throughout the operation. The paper is structured as follows: In Section 2, the system description, the control problems, and the critical aspects of system modeling are given. Consequently, the fundamental concept of active damping and the proposed active damping method are introduced in the same section. To validate the theoretical concept, the comparative studies between the proposed scheme and different AD methods are conducted and further discussed in Section 3. Finally, Section 4 presents the conclusion of this work.

\section{RESEARCH METHOD}

\subsection{The modelling of finite-control-set model predictive control of voltage source inverter with $L C L$} filter

As its name implies, MPC relies heavily on the precision of the system model. Therefore, proper system modeling and discretization are the critical elements that decide the control system's performance. Hence, a detailed explanation of system modeling is presented in this subsection. Figure 1 shows the circuit configuration of a voltage source inverter (VSI) with a $L C L$ output filter. The current through the inverterside filter inductance $L_{1}$ and the corresponding filter resistance $R_{1}$ filter current is represented with $\boldsymbol{i}_{f}=$ $\left[i_{f, \alpha} i_{f, \beta}\right]^{T}$. The current $\boldsymbol{i}_{o}=\left[i_{o, \alpha} i_{o, \beta}\right]^{T}$ symbolizes the output current flew through the grid-side inductance $L_{g}$. Additionally, the voltages $\boldsymbol{v}_{c}=\left[v_{c, \alpha} v_{c, \beta}\right]^{T}$ and $\boldsymbol{v}_{i}=\left[v_{i, \alpha} v_{i, \beta}\right]^{T}$ describe the output voltage measured across the filter capacitance and the internal inverter voltage, respectively. Finally, the voltage $v_{g}=\left[v_{g, \alpha} v_{g, \beta}\right]^{T}$ represents the grid voltage. For a balanced three-phase system, the zero sequence components can be neglected. Hence, all generic three-phase variables are converted into $\alpha \beta$-frame with the simplified, amplitude-invariant Clarke transformation. Thus, the model of a three-phase inverter with a $L C L$ filter is described in the $\alpha \beta$-frame with the capacitor dynamics equation (1) and the inductance dynamics equation (2). 
$C \frac{d v_{c}}{d t}=\boldsymbol{i}_{f}-\boldsymbol{i}_{o}$

$L_{1} \frac{d i_{f}}{d t}=\boldsymbol{v}_{i}-\boldsymbol{v}_{c}$

The system model based on (1) and (2) can be rewritten in the state-space form with the (3):

$$
\frac{d x}{d t}=A x+B v_{i}-B_{d} i_{o}
$$

where $\boldsymbol{x}=\left[\begin{array}{c}i_{f, \alpha} \\ i_{f, \beta} \\ v_{c, \alpha} \\ v_{c, \beta}\end{array}\right], \boldsymbol{A}=\left[\begin{array}{cccc}0 & 0 & -\frac{1}{L_{1}} & 0 \\ 0 & 0 & 0 & -\frac{1}{L_{1}} \\ \frac{1}{C} & 0 & 0 & 0 \\ 0 & \frac{1}{C} & 0 & 0\end{array}\right], \boldsymbol{B}=\left[\begin{array}{cc}\frac{1}{L_{1}} & 0 \\ 0 & \frac{1}{L_{1}} \\ 0 & 0 \\ 0 & 0\end{array}\right]$ and $\boldsymbol{B}_{\boldsymbol{d}}=\left[\begin{array}{cc}0 & 0 \\ 0 & 0 \\ -\frac{1}{C} & 0 \\ 0 & -\frac{1}{C}\end{array}\right]$.

As described by [8], a discrete-time model of the system derived from (3) for a sampling time $T_{S}$ can be expressed in (4).

$$
x(k+1)=A_{q} x(k)+B_{q} v_{i}(k)+B_{d q} i_{o}(k)
$$

where $\boldsymbol{A}_{q}=e^{\boldsymbol{A} T_{s}}, \boldsymbol{B}_{q}=\int_{0}^{T_{S}} e^{\boldsymbol{A} \tau} \boldsymbol{B} d \tau$ and $\boldsymbol{B}_{d q}=\int_{0}^{T_{S}} e^{\boldsymbol{A} \tau} \boldsymbol{B}_{d} d \tau$.

From (3)-(4), it shows that the future values $\boldsymbol{v}_{c}(k+1)$ and $\boldsymbol{i}_{f}(k+1)$ can be discovered following possible control set of input voltage $\boldsymbol{v}_{\boldsymbol{i}}(k)$. Nevertheless, the applications of FCS-MPC methods [9]-[12] conventionally only focus on the control of $\boldsymbol{i}_{f}(k+1)$, without considering the regulation of $\boldsymbol{v}_{c}(k+1)$. However, such control methods are vulnerable to the filter resonance and current distortion, as explained in the following section.

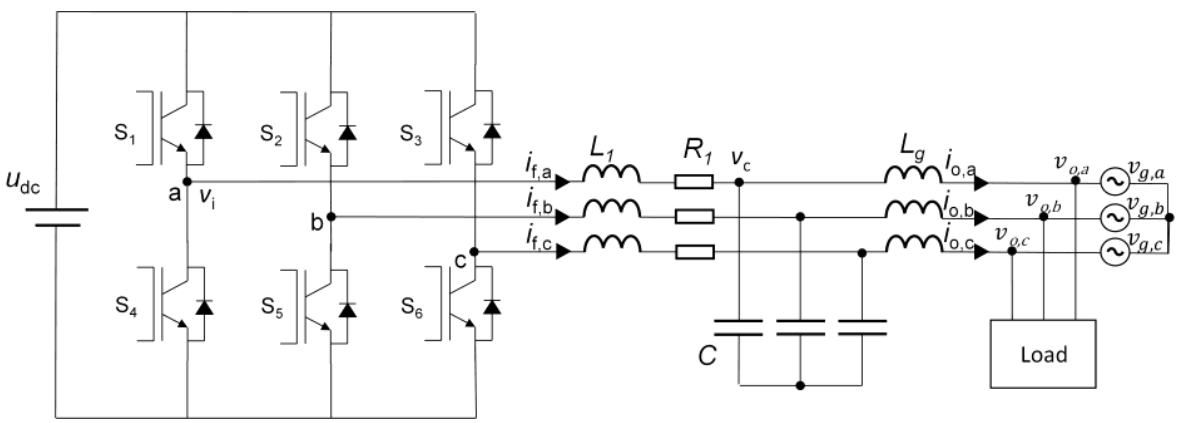

Figure 1. The power configuration of a grid-connected three-phase VSI with an LCL filter

\subsection{Control Problem: filter resonance}

The main task of the DG is to control the amount of active and reactive power, which is feeding into or/and out of the power grid. Following this, the $L C L$ filter is included in the system to improve the quality of the DG current that is proportional to these powers. However, the third-order system of the $L C L$ filter means we need to consider the presence of the resonance frequency described by the relationship (5):

$$
f_{\text {res }}=\frac{1}{2 \pi} \sqrt{\frac{1}{L_{1} C}}
$$

As mentioned in the previous section, most of the FCS-MPC-based grid-connected DG in the literature employs the predictive direct current control or predictive power control strategies [9]-[11] and only $i_{f}(s)$ is directly governed. To mitigate resonance, the other states of the filter, such as $v_{c}(s)$ and $i_{o}(s)$ needs to be indirectly regulated through the control of $i_{f}(s)$. This can be realized, as shown in Figure 2, by the so-called virtual resistance (VR) active damping method, which has been deployed in the FCS-MPC- 
based grid-connected VSI with some success [19]-[21]. However, problems might arise as soon as $v_{c}(s)$, is distorted by harmonics, which cannot be eliminated by the VR method.

Nevertheless, as mentioned previously, the resonance occurs between $L_{1}$ and $C$, because only $i_{f}(s)$ is controlled directly, whereas two other states, i.e., $i_{o}(s)$ and $v_{c}(s)$, are indirectly controlled and, hence, strongly reliant on filter impedances. Therefore, if we can control, $i_{o}(s)$ and $v_{c}(s)$, directly and simultaneously, we can reduce the level of dependency of $i_{o}(s)$ and $v_{c}(s)$ on filter impedances. With the proposed control scheme, we achieve this by utilizing the FCS-MPC scheme's multivariable control of both voltage and current of the $L C L$-filter simultaneously, thus restraining the resonant energy oscillation between the inductors and the capacitors. This idea was successfully applied to the grid-connected FCS-MPC-based AC/DC converter [22]-[24]. Later in the paper, we illustrate and compare two control methods (the VR-AD and the multivariable control AD methods) regarding their abilities to subdue the $L C L$ filter's resonance. The description of each of the control methods includes simulation results carried out in the PSCAD environment are also presented.

\subsection{Conventional virtual resistance active damping method}

Additional resistances are usually enclosed within the system for the prevention of resonant effects in the grid-side current. However, adding resistive units to the filter generate further losses. A well-known approach to avoid these losses is to utilize a VR-concept. This method simulates a damping resistance $R_{d}$ by injecting a virtual inverter-side resistive current component [16] and [19]-[21]. In this work, we implemented the control scheme with the VR-AD method based on the model predictive direct power control with AD ability proposed in [16]. Although different damping resistance realizations are possible, we chose only to insert the virtual $R_{d}$ in parallel to the filter capacitance due to a limited calculation burden. This is realized by injecting an additional current reference derived from the capacitor voltage's additional feedback, as shown in the block diagram of the VR-AD approach in Figure 2(a). It should be remembered, however, that if the distortion of the grid voltage is induced by harmonics, which cannot be eliminated by the VR-AD, those harmonics will be perpetually fed back to the current reference resulting in increased distortion of the gridside current waveforms. The transfer functions between the grid and the inverter currents, considering the passive damping shown in Figure 2(b) and2(c). The relationship between the damping factor $k_{d}$ and the parallel resistor $R_{d}$ can be found in [20] and $f_{\text {res }}$ is given by $k_{d}=1 / R_{d}$. The determining procedure of $k_{d}$ is well explained in [20] and thus will be omitted from this work.

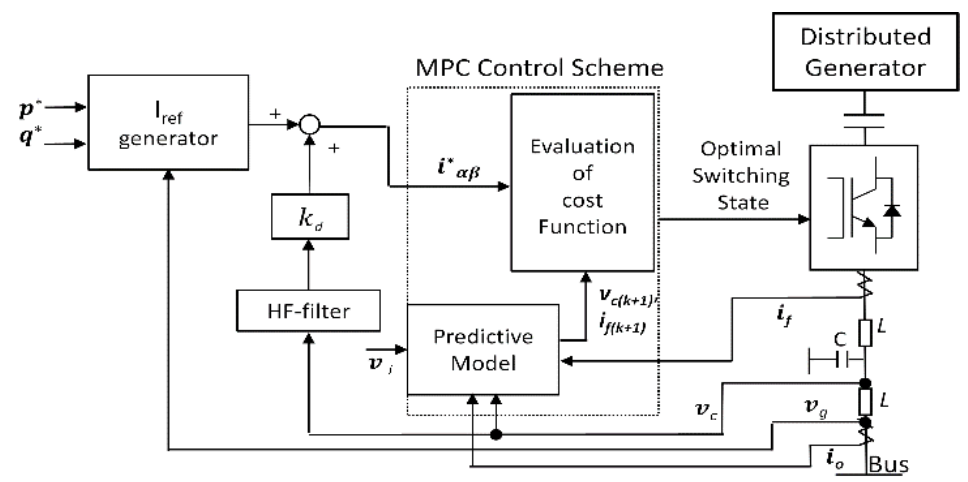

(a)

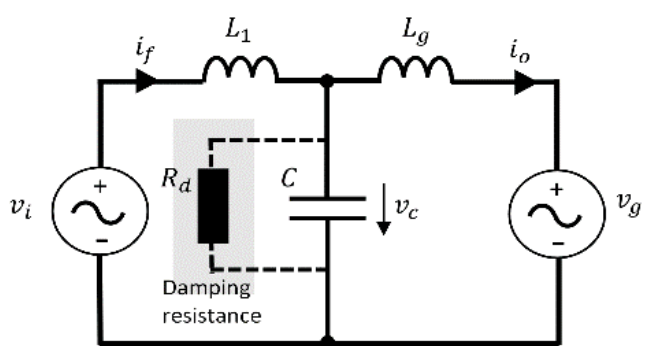

(b)

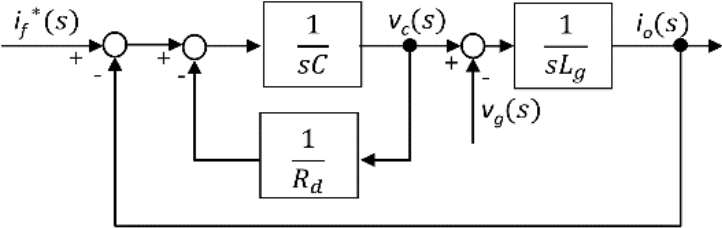

(c)

Figure 2. (a) Equivalent circuit and (b) control diagram of the VR-AD method for the VSI with $L C L$ output filters, (c) the FCS-MPC diagram with the VR-AD method 


\subsection{The proposed multivariable control active damping method}

The proposed FCS-MPC utilizes a multiple-inputs-multiple-outputs (MIMO) system to control both $v_{c}(s)$ and $i_{f}(s)$ simultaneously as illustrated by the control diagram in Figure 3. The multivariable control of FCS-MPC is carried out with the same bandwidth. This ascertains that the regulation of $v_{c}(s)$ can dampen the distortion caused by the harmonics of $i_{f}(s)$ and the control of $i_{f}(s)$ can reduce the disturbance caused by the harmonics of $v_{c}(s)$. This approach is fundamentally different from the traditional cascade control with outer loop voltage and inner loop current control, where the dynamic response of the inner loop has to be substantially higher than its outer loop counterpart. Therefore, the outer loop's control is not quick enough to provide a damping effect when both $v_{c}(s)$ and $i_{f}(s)$ are oscillating with the resonant frequency. Additionally, only two degrees of liberation prevail out of the three states of the $L C L$-filter since $i_{o}(s)$ is a function of $v_{c}(s)$ and $i_{f}(s)$. Hence, by controlling both degrees of freedom of the $L C L$-filter, the filter's frequency response is no longer highly dependent on the filter impedance. The unregulated oscillation between the inductors and the capacitors is thus effectively mitigated. Since both the filter current and the capacitor voltage will be controlled in this approach, we will refer to this AD method as the multivariable control AD method Moreover, considering the control diagram in Figure 2(c), it is shown that the oscillation in the filter occurs because the harmonic contents in the $i_{f}(s)$ is amplified by the impedance of the capacitor, and similarly, the harmonic contents in $v_{c}(s)$ is influenced by the filter impedance. Thus, the oscillation caused by the $L C L$ filter resonance can be damped by merely reducing the harmonics induces distortion in $i_{f}(s)$ and $v_{c}(s)$. Since the control of the FCS-MPC occurs in real-time and in $\alpha \beta$-frame, if we can ensure that the inverter's side filter current and the capacitor voltage always track sinusoidal command waveforms, it will fortify the active damping ability of the control scheme. In order to achieve this, we insert a function proposed in [25], referred to as second-order generalized integrator (SOGI) bandpass filter, to the current and voltage command, as shown in Figure 3. Only the content at the fundamental frequency remains in the current and voltage command, and the oscillation caused by the filter resonance is suppressed with this function. As illustrated in Figure 3, we chose to employ the virtual synchronous generator (VSG) in this work to obtain the voltage command. In contrast, the current command is derived from the voltage command and the real-time system's variables by imitating the relationship between the inverter voltage, filter currents, and the capacitor voltage. The VSG control scheme can regulate the inverter's output active and reactive powers by manipulating the output voltage. Hence, it is suitable for the proposed control scheme for DG application, where proper power control and multivariable control of voltage and current are desired. Additionally, with the output voltage and current regulation, the VSI can operate in both grid-connected and stand-alone operations. The concept of VSG is derived from the well-known swing equation (6).

$$
P_{\text {in }}-P_{\text {out }}=J \omega_{m} \frac{d \omega_{m}}{d t}+D\left(\omega_{m}-\omega_{g}\right)
$$

where $P_{\text {in }}$ is virtual shaft power, $P_{\text {out }}$ and $Q_{\text {out }}$ are the active and reactive power of the measured output. $\omega_{m}$ is virtual rotor angular frequency. $\omega_{g}$ is output voltage angular frequency, and J, D are virtual inertia and virtual damping factor, respectively. The VSG control scheme is adopted from [14], and since the VSG control is not the focus of this work, its explanation will be omitted.

As mentioned earlier, the current reference can be obtained by imitating the connection between the inverter voltage, filter currents, and the capacitor voltage, shown in Figure 1, which can be represented in a stationary frame $(\alpha \beta)$ with (7).

$$
\boldsymbol{v}_{i, \alpha \beta}=\boldsymbol{v}_{c, \alpha \beta}+\boldsymbol{i}_{f, \alpha \beta}\left(R_{f}+j\left(X_{L, f}+X_{S}\right)\right)
$$

Where $\boldsymbol{v}_{i, \alpha \beta}$ is inverter voltage, $\boldsymbol{i}_{f, \alpha \beta}$ symbolizes the filter current and $\boldsymbol{v}_{c, \alpha \beta}$ represents the capacitor voltages. Let $X_{S}$ indicate the virtual stator reactance, $X_{L, f}$ indicate the filter reactance and $R_{f}$ symbolize filter resistance. If we equalize the $\boldsymbol{v}_{i, \alpha \beta}$ in (7) to the voltage command of the VSG control, the currents command $i_{\alpha}^{*}$ and $i_{\beta}^{*}$ can be described as (8)

$$
\left[\begin{array}{l}
i_{\alpha}^{*} \\
i_{\beta}^{*}
\end{array}\right]=\boldsymbol{Y}\left\{\left[\begin{array}{l}
E_{0} \cos \theta_{m} \\
E_{0} \sin \theta_{m}
\end{array}\right]-\left[\begin{array}{l}
v_{c, \alpha} \\
v_{c, \beta}
\end{array}\right]\right\}
$$


Where $\boldsymbol{Y}=\frac{1}{R_{f}^{2}+X_{S}{ }^{2}}\left[\begin{array}{cc}R_{f} & X_{S} \\ -X_{S} & R_{f}\end{array}\right], E_{0}$ represents the internal emf command and $\theta_{m}$ the phase angle command from the VSG control. In the proposed FCS-MPC, the control algorithm is set to follow the voltage and current command $\left(\boldsymbol{v}_{\alpha \beta}^{*}\right.$ and $\left.\boldsymbol{i}_{\alpha \beta}^{*}\right)$ simultaneously. This is realized by defining the cost function (9).

$$
g=k_{v}\left(\boldsymbol{v}_{c, \alpha \beta(\mathrm{k}+1), \mathrm{pu}}-\boldsymbol{v}_{\alpha \beta, \mathrm{pu}}^{*}\right)^{2}+k_{i}\left(\boldsymbol{i}_{f, \alpha \beta(\mathrm{k}+1), \mathrm{pu}}-\boldsymbol{i}_{\alpha \beta, \mathrm{pu}}^{*}\right)^{2}
$$

Where $\boldsymbol{v}_{\alpha \beta, p u}^{*}=\left[v^{*}{ }_{\alpha} v_{\beta}^{*}\right]^{T}$ is the voltage command in the per-unit system, $\boldsymbol{i}_{\alpha \beta, p u}^{*}=\left[\boldsymbol{i}_{\alpha}^{*} \boldsymbol{i}_{\beta}^{*}\right]^{T}$ is the current command in the per-unit system, $\boldsymbol{v}_{c, \alpha \beta}(k+1)=\left[v_{\alpha} v_{\beta}\right]^{T}$ symbolizes the predicted filter capacitor voltage and $\boldsymbol{i}_{f, \alpha \beta}(k+1)=\left[i_{\alpha} i_{\beta}\right]^{T}$ represents the predicted filter current. Furthermore, weighting factors $k_{i}$ and $k_{v}$ are included to customize the cost between the voltage and current controls in the cost function.

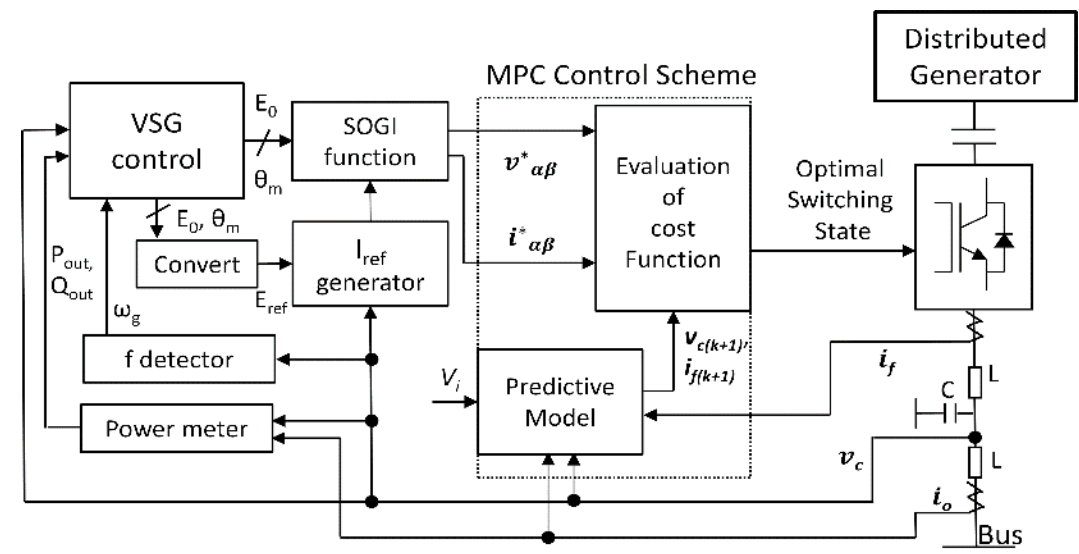

Figure 3. The control diagram of the proposed multivariable control AD scheme for the VSI-based DG system

\section{RESULTS AND DISCUSSION}

To study the filter resonance effect and evaluate the proposed AD method's performance, we conducted the test circuit's simulation study depicted in Figure 1 in the PSCAD/EMTDC environment. The simulation study is conducted under three different cases. In case 1, the $L C$ and $L C L$ filter's effects on the DG system are first studied. The differences between the voltage and current waveforms are observed when either the $L C$ or $L C L$ filters are used. In these cases, no active damping approach is deployed in the control scheme, meaning the $k_{v}$ of the cost function of the FCS-MPC is set to 0 , while the $k_{i}$ is set at 1 . In this condition, only the output current $\left(\boldsymbol{i}_{o}\right)$ is controlled through the control of inverter current $\left(\boldsymbol{i}_{\text {inv }}\right)$, and the filter voltage is uncontrolled. The current command is obtained directly from $\boldsymbol{v}_{g}$ and the instantaneous active and reactive power equations. It is noteworthy that the power command changing is not conducted in this test. In case 2 , similar to case 1 , the control scheme is set to regulate only $\boldsymbol{i}_{o}$. However, the virtual resistive current generated by the VR method is included in the current command to provide the system with an AD ability. For case 3, the ratio of $k_{v}: k_{i}$ is set to $1: 1$ to achieve the simultaneous control of the filter voltage and the output current. Then we observed the two AD approaches' effectiveness in suppressing the oscillation caused by the $L C L$ filter resonance. Furthermore, to study the AD methods' robustness under grid voltage disturbances, additional tests were conducted for case 2 and case 3 with a $6^{\text {th }}$ order harmonic current contents injecting to the grid voltage. The harmonic content's size was set to $2 \mathrm{~A}(0.08 \mathrm{pu})$ and $4 \mathrm{~A}(0.16 \mathrm{pu})$, respectively. The DG system was operating in grid-connected mode. In cases 1 and 2, it is set to operate with nominal output power $P_{0}$ equal to $5 \mathrm{~kW}$ and the nominal reactive output power $Q_{0}$ is set at 0 Var. To observe the effect of the dynamic response of the control schemes, the nominal output power $P_{0}$ is changed to $3.5 \mathrm{~kW}$ at $\mathrm{T}=4 \mathrm{~s}$. In case 3 , the system was operating with the reference active power obtained from the VSG control. The VSG control utilizes the equation of $P_{\text {ref }}=P_{0}-k_{p}\left(\omega_{\text {grid }}-\omega_{\text {base }}\right)$, where the calculated grid 
Frequency is $\omega_{\text {grid }}$, the grid nominal frequency is $\omega_{\text {base }}$, which is fixed at $376.99 \mathrm{rad} / \mathrm{s}$, and the frequency droop gain $k_{p}$ is selected at $20 \mathrm{pu}$, for which nominal output power $P_{0}$ is set at $5 \mathrm{~kW}$. The nominal reactive output power $Q_{0}$ is set at 0 Var. Also, in this case, $P_{0}$ changed to $3.5 \mathrm{~kW}$ at T $=4 \mathrm{~s}$. The sampling frequency $f_{s, v s i}$ is selected at $30 \mathrm{kHz}$ for all three cases, which generates an average switching frequency of around $15 \mathrm{kHz}$ in the DG system. The essential parameters of the simulation study are given in Table 1.

Table 1. The parameters used in the test circuits.

\begin{tabular}{cccc}
\hline Parameters & Values & Parameters & Values \\
\hline$S_{\text {base }}$ & $5 \mathrm{kVA}$ & $L_{1}$ & $2.5 \mathrm{mH}$ \\
$L_{g}$ & $0.4 \mathrm{mH}$ & $C$ & $10 \mu \mathrm{F}$ \\
$U_{d c}$ & $400 \mathrm{~V}$ & $f_{s, v s i}$ & $30 \mathrm{kHz}$ \\
$V_{\text {grid }}$ & $200 \mathrm{~V}$ & $R_{d}$ & $16 \Omega$ \\
\hline
\end{tabular}

\subsection{Effect of $L C L$ filters on the FCS-MPC-based DG system without AD methods}

Without AD methods, the DG's voltage and current waveforms are illustrated in Figure 4 (a) when $L C$ filters are deployed between the VSI and the grid. It displayed that the current-controlled FCS-MPC can achieve a sinusoidal grid current. However, as shown in Figure 4 (b), when we utilize $L C L$ filters in the system instead of the $L C$ filter, the DG's voltage and current waveforms suffer from resonance and distortion. It verifies that $\mathrm{AD}$ methods are required for FCS-MPC-based DG when $L C L$ filters are included in the system. In case $L C$ filters are utilized, the filter current THD amount to $2 \%$, while the filter current THD is as high as $6 \%$ when $L C L$ filters are deployed. The collection of current THD of all cases is presented in Table 2 .
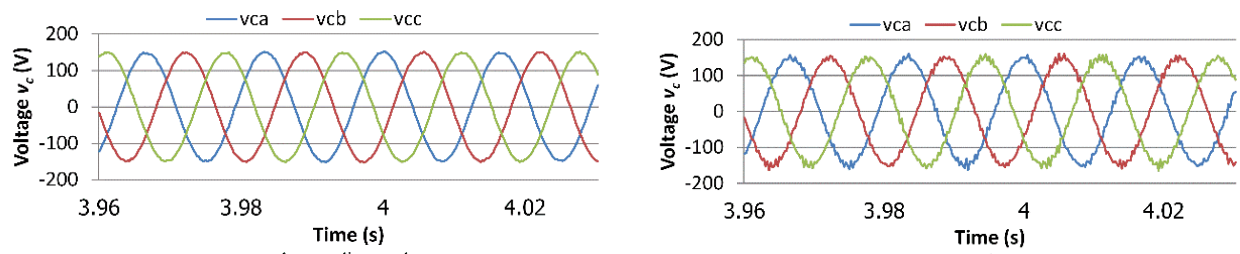

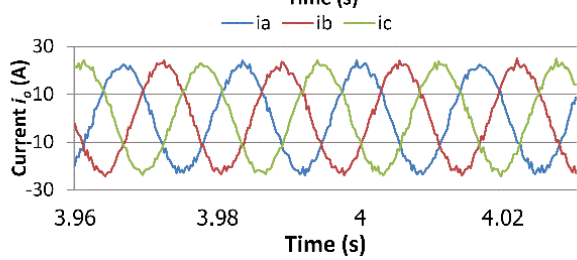

(a)

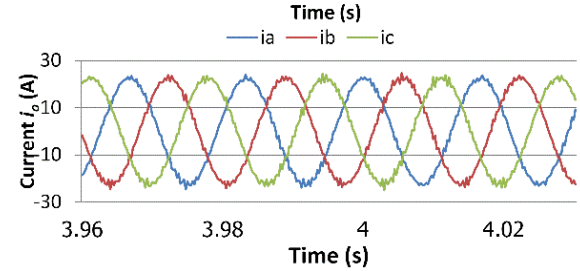

(b)

Figure 4. Voltage $v_{c}$ and current $i_{o}$ waveforms of the DG system without AD methods, (a) when $L C$ filters are deployed. (b) when $L C L$ filters are deployed

\subsection{The conventional VR-AD method}

In case 2, the VR-AD method is included in the control scheme. The voltage and current waveforms of the DG system are illustrated in Figure 5 (a). It clearly shows that the $L C L$ filter resonance distortion significantly reduced thanks to the VR method's employment. As shown in Table 2, the current THD of this case amounts to $2.75 \%$, significantly lower than the $6 \%$ THD when no AD approach is deployed. The output active and reactive power is also included in Figure 5 (a) to verify that the control scheme has proper control over the output power. The control's dynamic response is rapid, as seen when the power command changes at $\mathrm{T}=4 \mathrm{~s}$. Moreover, to evaluate the AD ability's performance, we purposely create an additional $0.08 \mathrm{pu}$ harmonic content near the resonant frequency. However, as discussed earlier, the harmonics caused by grid voltage distortion are unendingly fed back to the current reference resulting in increased distortion of the current waveforms. The voltage and current waveforms of the DG system during grid disturbances are illustrated in Figure 5 (b). As concluded in Table 2, the THD of the current increases to $6.5 \%$ when a $0.08 \mathrm{pu}$ 6 th harmonic contents distort the grid voltage, and the THD is as high as $12 \%$ when a $0.16 \mathrm{pu} \mathrm{A}$ harmonic contents distort the grid voltage. 

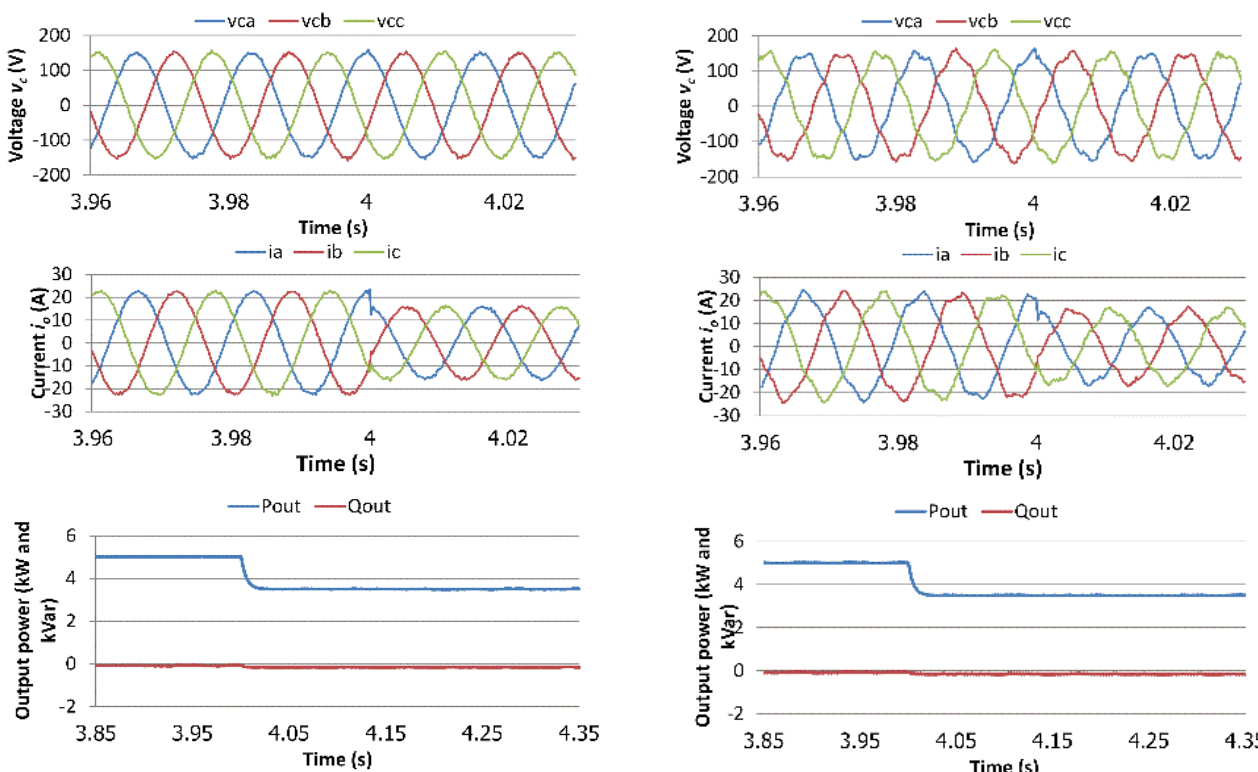

(a)

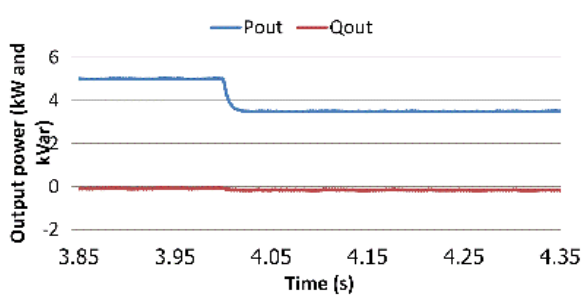

(b)

Figure 5. Voltage $v_{c}$ and current $i_{o}$ waveforms of the DG system with $L C L$ filters using the VR-AD method (a) without additional harmonic contents, (b) with additional harmonic contents

Table 2. The collection of the current THDs for the test study

\begin{tabular}{ccccc}
\hline AD types & LC & $L C L$ & $\begin{array}{c}L C L \text { with } 0.08 \text { pu harmonic } \\
\text { current contents }\end{array}$ & $\begin{array}{c}L C L \text { with } 0.16 \text { pu } \\
\text { harmonic current contents }\end{array}$ \\
\hline No AD & 2 & 6 & - & - \\
VR-AD & - & 2.75 & 6.5 & 12 \\
Multivariable control AD & - & 3 & 4.5 & 6.5 \\
\hline
\end{tabular}

\subsection{The proposed multivariable AD method}

In case 3, the proposed multivariable control $\mathrm{AD}$ method is included in the control scheme. The voltage and current waveforms of the DG system are illustrated in Figure 6 (a). Similar to case 2, the $L C L$ filter resonance distortion significantly reduced thanks to the proposed AD method's employment. The output active and reactive powers are also correctly managed, as illustrated in Figure 6 (a). Although it shows a slower dynamic response when the power command is changed compared to the direct power control, the settling time is within $0.2 \mathrm{~s}$, which is within an acceptable range for the DG application. The result of the current THD collected in Table 2 shown that the proposed control has similar AD performance compared with the VR method. The voltage and current waveforms of the DG system illustrated in Figure 6 (b) also shown that the grid current distortion can be efficiently suppressed when an additional 0.08 pu 6th harmonic content is added to the grid. In this case, the proposed control has a superior AD performance compared with the VR method, as the current THD amounts to $4.5 \%$ compared with the $6.5 \%$ THD of the VR method. The difference becomes more apparent when $0.16 \mathrm{pu}$ 6th harmonic content distorted the grid voltage, as the current THD amounts to $6.5 \%$ compared with the $12 \%$ THD of the VR method. This trend continues even when a higher amount of harmonic contents exist in the grid voltage. However, results with higher harmonics contents might not be realistic. Thus, they are excluded from this study. Nevertheless, the results indicate that the proposed control scheme is more robust under grid voltage disturbances than the existing method.

Furthermore, the proposed control offers more flexibility than the VR method since the proposed AD approach only uses the multi-control of filter states to damp the filter resonance without any additional command. Hence, other parts of the control algorithms are not influenced by the AD approaches. For example, the proposed method's power control is not affected by the added current command part, unlike in the VR method, where the effect of VR current to the power command needs to be compensated. It is also easy to integrate additional control algorithms such as the VSG control to the multivariable control. This enables the proposed control to operate in both grid-connected and stand-alone modes, making it a suitable control algorithm for DG applications. 

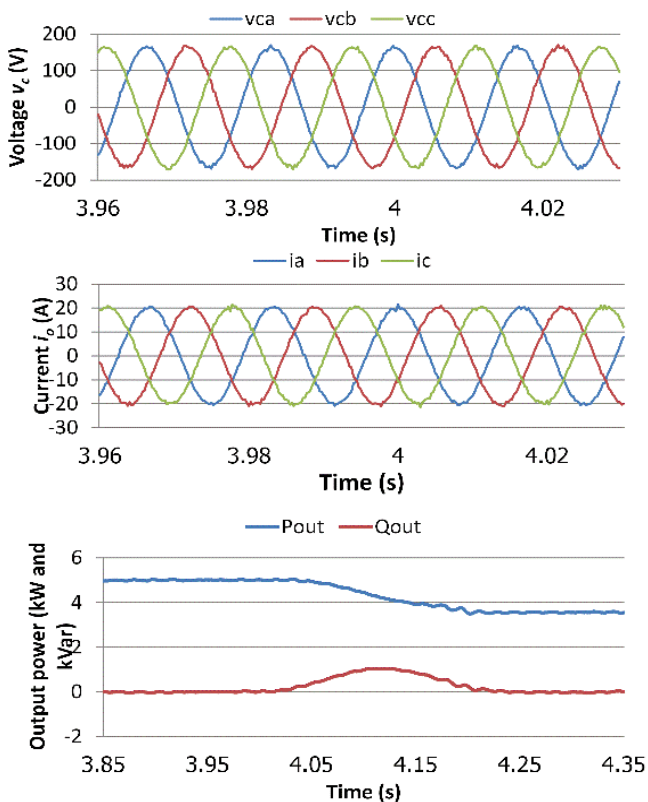

(a)

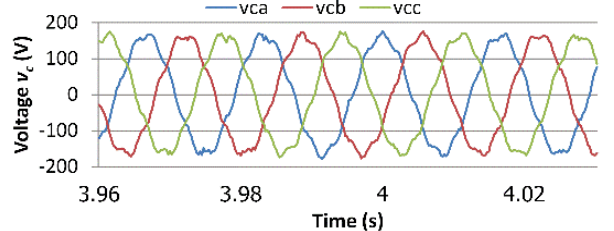

-ia $\frac{\text { Time }(s)}{-i b}$ ic
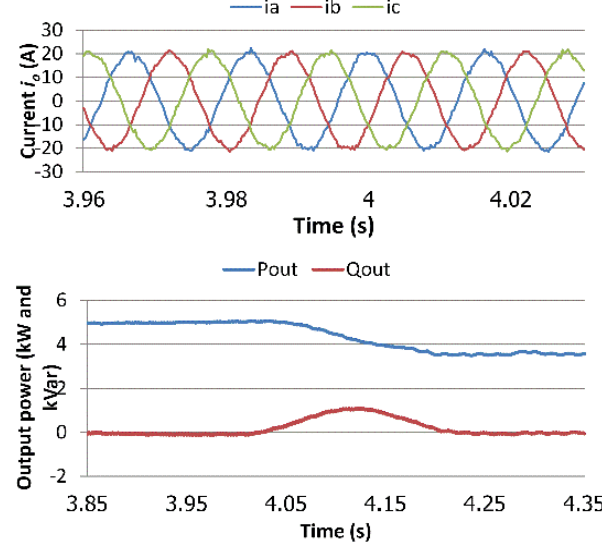

(b)

Figure 6. Voltage $v_{c}$ and current $i_{o}$ waveforms of the DG system with $L C L$ filters using the proposed AD method; (a) without additional harmonic contents; (b) with additional harmonic contents

\section{CONCLUSION}

In this paper, a FCS-MPC-based active damping method has been proposed and compared with a virtual resistance active damping algorithm, applying in a predictive control scheme. The study shows that $L C L$ filter resonance oscillations were effectively damped using the novel FCS-MPC-based multivariable control algorithm. Simulation results confirmed the proposed method's capability to eliminate lower-order grid current harmonics and decrease sensitivity to grid voltage distortion. In the steady-state, the proposed method's performance in damping the oscillation is comparable to the existing method. When the grid is suffering from voltage distortion, the proposed method showed significant AD performance improvements compared to the existing method. Additionally, with the multivariable control of voltage and current, the proposed control system can also operate in both grid-tied and stand-alone operations. Finally, thanks to the flexibility and ease of integrating with different control algorithms, the proposed multivariable FCS-MPCbased $\mathrm{AD}$ method offers excellent control performance and can be considered a serious alternative to the classical PWM-based AD method. The present work was an initial step, verifying the effectiveness of applying a multivariable control AD method in a VSI-based DG, and for future steps of this work, conducting experimental tests to evaluate and validate the proposed control strategy is intended.

\section{REFERENCES}

[1] Solarpowereurope.org. "Global Market Outlook for Solar Power/2016-2020,".2020 [Online]. Available: https://www.solarpowereurope.org. [Accessed: 23- Sep- 2020].

[2] Haval Sardar Kamil, Dalila Mat Said, Mohd Wazir Mustafa, Mohammad Reza Miveh and Nasarudin Ahmad. "Low-voltage Ride-through Methods for Grid-connected Photovoltaic Systemsin Microgrids: A Review and Future Prospect." in International Journal of Power Electronics and Drive Systems (IJPEDS), vol, 9, no. 4, pp. 1834-1841, Dec 2018.

[3] Ihsan Jabbar Hasan, Nahla Abdul Jalil Salih and Nadhir Ibrahim Abdulkhaleq. "Three-phase photovoltaic grid inverter system design based on PIC24FJ256GB110 for distributed generation." in International Journal of Power Electronics and Drive Systems (IJPEDS), vol, 10, no. 3, pp. 1215-1222, Sep 2019.

[4] Aya M. Elsherbiny, Adel S. Nada and Mohammed Kamal. "Smooth transition from grid to stand-alone solar diesel mode hybrid generation system with a battery." in International Journal of Power Electronics and Drive Systems (IJPEDS), vol, 10, no. 4, pp. 2065-2075, Dec 2019.

[5] J. Rodriguez et al., "State of the Art of Finite Control Set Model Predictive Control in Power Electronics," in IEEE Transactions on Industrial Informatics, vol. 9, no. 2, pp. 1003-1016, May 2013. 
[6] Mai Van Chung, Do Tuan Anh, Phuong Vu, and Manh Linh Nguyen. "Hardware in the loop co-simulation of finite set-model predictive control using FPGA for a three-level CHB inverter." in International Journal of Power Electronics and Drive Systems (IJPEDS), vol, 11, no. 4, pp. 1719-1730, Dec 2020.

[7] Najmeh Movahhed Neya, Sajad Saberi, and Babak Mozafari. "Direct predictive speed control of permanent magnet synchronous motor fed by matrix converter." in International Journal of Power Electronics and Drive Systems (IJPEDS), vol. 11, no. 4. pp. 1719-1730, Dec 2020.

[8] P. Cortes, G. Ortiz, J. I. Yuz, J. Rodriguez, S. Vazquez, and L. G. Franquelo, "Model Predictive Control of an Inverter with Output LC Filter for UPS Applications," in IEEE Transactions on Industrial Electronics, vol. 56, no. 6, pp. 1875-1883, Jun 2009.

[9] F. Donoso, A. Mora, R. Cárdenas, A. Angulo, D. Sáez and M. Rivera, "Finite-Set Model-Predictive Control Strategies for a 3L-NPC Inverter Operating with Fixed Switching Frequency," in IEEE Transactions on Industrial Electronics, vol. 65, no. 5, pp. 3954-3965, May 2018.

[10] B. Arif, L. Tarisciotti, P. Zanchetta, J. C. Clare and M. Degano, "Grid Parameter Estimation Using Model Predictive Direct Power Control," in IEEE Transactions on Industry Applications, vol. 51, no. 6, pp. 4614-4622, Nov.-Dec. 2015

[11] J. Hu, J. Zhu, D. G. Dorrell, "Model predictive control of inverters for both islanded and grid-connected operations in renewable power generations," in IET Renewable Power Generation, vol. 8, Issue. 3, pp. 240-248, 2014.

[12] J. Scoltock, T. Geyer and U. K. Madawala, "A Model Predictive Direct Current Control Strategy with Predictive References for MV Grid-Connected Converters with LCL -Filters," in IEEE Transactions on Power Electronics, vol. 30, no. 10, pp. 5926-5937, Oct. 2015.

[13] J. Jongudomkarn, J. Liu and T. Ise, "Comparison of Current-Limiting Strategies of Virtual Synchronous Generator Control during Fault Ride-Through," in IFAC-PapersOnLine, vol. 51, issue 28, pp. 256-261, 2018.

[14] J. Jongudomkarn, J. Liu and T. Ise, "Virtual Synchronous Generator Control with reliable Fault Ride-Through ability: A solution based on Finite-Set Model Predictive Control," in IEEE Journal of Emerging and Selected Topics in Power Electronics, vol. 8, Issue 4, pp. 3811 - 3824, 2020.

[15] J. Jongudomkarn, J. Liu, Y. Yanagisawa, H. Bevrani and T. Ise, "Model Predictive Control for Indirect Boost Matrix Converter Based on Virtual Synchronous Generator," in IEEE Access, vol. 8, pp. 60364-60381, 2020.

[16] A. Reznik, M. G. Simoes, A. Al-Durra, and S. M. Muyeen, "LCL filter design and performance analysis for gridinterconnected systems," in IEEE Transactions on Industry Applicatons., vol. 50, no. 2, pp. 1225-1232, Mar.-Apr. 2014.

[17] A. A. Rockhill, M. Liserre, R. Teodorescu, and P. Rodriguez, "Grid filter design for a multimegawatt mediumvoltage voltage-source inverter," in IEEE Transactions on Industrial Electronics, vol. 58, no. 4, pp. 1205-1217, Apr. 2011.

[18] Seung-Jin Yoon, Thanh Van Nguyen, and Kyeong-Hwa Kim. "Current control of grid-connected inverter using integral sliding mode control and resonant compensation," in International Journal of Power Electronics and Drive Systems (IJPEDS), vol. 10, no. 2, pp. 1022-1033, June 2019.

[19] J. Scoltock, T. Geyer, and U. Madawala, "Model predictive direct current control for a grid-connected converter: LCL filter versus 1-filter," in Proc. 2013 IEEE Int. Conf. Ind. Technol., pp. 576-58, 2013.

[20] L. A. Serpa, S. Ponnaluri, P. M. Barbosa and J. W. Kolar, "A Modified Direct Power Control Strategy Allowing the Connection of Three-Phase Inverters to the Grid Through LCL Filters," in IEEE Transactions on Industry Applications, vol. 43, no. 5, pp. 1388-1400, Sep-Oct. 2007.

[21] X. Zhang, Y. Wang, C. Yu, L. Guo, and R. Cao, "Hysteresis model predictive control for high-power gridconnected inverters with output LCL filter," in IEEE Transactions on Industrial Electronics, vol. 63, no. 1, pp. 246-256, Jan. 2016

[22] P. Falkowski and A. Sikorski, "Dead-time compensation in a new FCS-MPC of an AC/DC converter with a $L C L$ filter," 2016 13th Selected Issues of Electrical Engineering and Electronics (WZEE), Rzeszow, , pp. 1-6, 2016.

[23] N. Panten, N. Hoffmann, and F. W. Fuchs, "Finite Control Set Model Predictive Current Control for GridConnected Voltage-Source Converters With LCL Filters: A Study Based on Different State Feedbacks," in IEEE Transactions on Power Electronics, vol. 31, no. 7, pp. 5189-5200, July 2016.

[24] P. Falkowski and A. Sikorski, "Finite Control Set Model Predictive Control for Grid-Connected AC-DC Converters With LCL Filter," in IEEE Transactions on Industrial Electronics, vol. 65, no. 4, pp. 2844-2852, April 2018.

[25] A. V. Timbus, P. Rodriguez, R. Teodorescu, M. Liserre, and F. Blaabjerg, "Control Strategies for Distributed Power Generation Systems Operating on Faulty Grid," 2006 IEEE International Symposium on Industrial Electronics, Montreal, Que., pp. 1601-1607, 2006.

\section{BIOGRAPHIES OF AUTHORS}




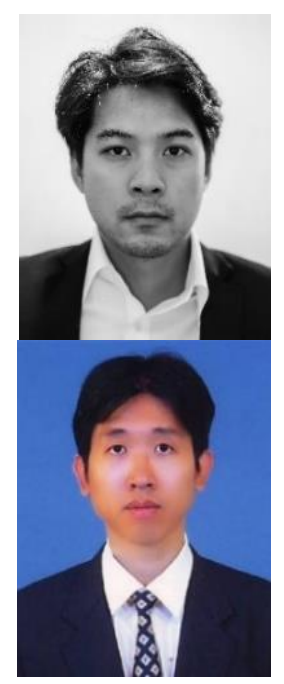

Jonggrist Jongudomkarn received the B.Eng. and Diplom-Ingenieur Univ. (M.Sc.) degrees in electrical engineering and information technology from the Technical University of Munich, Germany, in 2012 and 2013, respectively. He then received the Ph.D. degree in electrical engineering from Osaka University, Japan, in 2020.

He was with the Faculty of Engineering, Khon Kaen University in Thailand from 2014 to 2016 and is currently working at the Faculty of Engineering, Khon Kaen University in Thailand. His research interests include distributed generators, power quality, electric machine drives, and power conversion.

Warayut Kampeerawat received the B.Eng. and M.Eng. degrees in electrical engineering from Khon Kaen University, Thailand, in 2005 and 2007, respectively. He then received the Ph.D. degree in electrical engineering and information systems from the University of Tokyo, Japan, in 2019.

He was with the Faculty of Engineering, Khon Kaen University from 2012 and is currently an assistant professor at the Faculty of Engineering, Khon Kaen University in Thailand. His research interests include optimization techniques applied to power system and electric railway, power and energy management, power system protection, and power quality. 